\title{
Decision-Making in Rats is Sensitive to Rare and Extreme Events: the Black Swan Avoidance*
}

\author{
Mickaël Degoulet ${ }^{\dagger} \quad$ Louis-Matis Willem ${ }^{\ddagger} \quad$ Christelle Baunez $^{\S}$ \\ Stéphane Luchini₫ Patrick A. Pintus\|
}

November 1, 2021

Preliminary Draft not To Be Circulated Without the Authors' Permission

In Brief: Most studies assessing decision-making under uncertainty use events with probabilities that are above 10-20 \% . Here, to study decision-making in radical uncertainty conditions, Degoulet, Willem, Baunez, Luchini and Pintus provide a novel experimental design that aims at measuring the extent to which rats are sensitive - and how they respond - to extremely rare (below 1\% of probability) but extreme events in a four-armed bandit task. Gains (sugar pellets) and losses (time-out punishments) are such that large - but rare - values materialize or not depending on the option chosen. The results show that all rats diversify their choices across options. However, most rats exhibit sensitivity to rare and extreme events despite their sparse occurrence, by combining more often options with extreme gains (Jackpots) and/or avoidance of extreme losses (Black Swans). In general, most rats' choices feature one-sided sensitivity in favor of trying more often to avoid extreme losses than to seek extreme gains - that is, they feature Black Swan Avoidance.

\section{Highlights:}

- A novel experiment is proposed to measure whether rats are sensitive - and how they respond - to radical uncertainty materialized by extremely rare and extreme events in a four-armed bandit task;

- Most rats exhibit sensitivity to rare and extreme events;

- Most rats opt more often to avoid rare and extreme losses than to seek rare and extreme gains: they feature Black Swan avoidance.

\footnotetext{
*The authors would like to especially thank Raouf Boucekkine, Thibault Gajdos, Tobias Kalenscher, Drazen Prelec and Wolfram Schultz for very useful discussions and encouragement at early and more recent stages of this research, which has had a long gestation lag. First Draft: October 2021. This work was supported by the French National Research Agency Grant ANR-17-EURE-0020, and by the Excellence Initiative of Aix-Marseille University A*MIDEX.

$\dagger$ Institute of Neurosciences Timone, CNRS \& Aix-Marseille University. Email: mickael.degoulet@univ-amu.fr.

‡Ecole Centrale Marseille. Email: louis.willem@centrale-marseille.fr.

§Institute of Neurosciences Timone, CNRS \& Aix-Marseille University. Email: christelle.baunez@univ-amu.fr.

ฯ Aix-Marseille University, CNRS, AMSE. Email: stephane.luchini@univ-amu.fr.

"CNRS-InSHS and Aix-Marseille University, CNRS, AMSE. Email: patrick.pintus@univ-amu.fr.
} 


\section{Summary}

Exploration and exploitation are key drivers of behavior in animals and humans (see [10] for an overview). Although uncertainty is a central dimension in the related literature, most studies on decision-making rely on lotteries with frequencies that are typically larger than $10 \%$ (e.g. [3], [4], [15], [19]). However, many species experience events that are much rarer but nevertheless entail a potentially large impact when interacting with their environment, the extinction of large groups or species being an extreme example. To the extent that both their consequences and their frequencies are highly uncertain, because historical data either lacks or is scarce, such rare and extreme events (REE thereafter) are challenging for decision-making. Little is arguably known about whether animal species, including humans, are sensitive to - and how they cope with - such infrequent and consequential events when making choices. The present study thus aimed at designing experiments where rats interact repeatedly with their environment through a 4-armed bandit task, in which they face REE that occur with a frequency smaller than $1 \%{ }^{1}$

In addition to dealing with very low-probability events, the novelty of our experimental design is that it builds upon the notion that, in such a context, it might pay off to build exposures to uncertainty that limit the consequences of extremely "negative" risks - or losses - while leaving open the possibilities to benefit from "positive" and large risks - or gains (called "Anti-fragile" exposures in Taleb [18]). Such options are best thought of as being convex, because they combine accelerating and possibly large gains, together with decelerating and limited losses. Symmetrically, an option that rules out extreme gains (Jackpots thereafter) but may trigger very large losses (Black Swans) is concave in our design. The evolutionary perspective would ask whether convex (anti-fragile) choices are indeed preferred by animals and humans, and whether there are natural foundations for convex choices that are embodied in the brain and possibly relate to fitness. As argued by [8, page 1], "In order to fully comprehend the origins of human choice behavior, we should investigate the evolutionary roots of our decision-making processes by looking at choice mechanisms and their neural substrates in animals".

Because of our specific interest in REE on both sides, our framing integrates gains (sugar pellets) and losses (time-out punishment). One attractive feature of our design is that it provides two direct measures that help interpreting our behavioral data. First, Total Sensitivity to Rare and Extreme Events measures the extent to which rats seek large gains and avoid large losses. Second, One-sided Sensitivity to Rare and Extreme Events captures seeking large gains is favored compared to avoiding large losses, with Black Swan Avoidance and Jackpot Seeking as limiting cases. Using our setup on a sample of 20 rats, with about 6000 stimuli per rat, we document two sets of results. First, all rats diversify their choices across a set of options, primarily 2 out of the 4 available. Most rats (19 out of 20) exhibit moderate to high levels of Total Sensitivity. This means that most rats choose to diversify their choices across options that more often ensure extreme gains and/or limited losses than limited gains and/or extreme losses. In addition, most rats (13 out of 20) tend to exhibit Black Swan Avoidance: they have high Total Sensitivity and favor avoiding Black Swans over seeking Jackpots. We interpret such a behavior as significant uncertainty aversion towards rare and extreme losses. Consistent with such an interpretation, we also find that Total Sensitivity and Black Swan Avoidance are reinforced after a rare and extreme loss is experienced.

\footnotetext{
${ }^{1}$ Following the design of the Iowa Gambling Task for human subjects (see [1]), similar tasks for rodents typically assume that the less frequent events have probability values that are about or larger than $10 \%$ (see [12] and [20]).
} 
Figure 1: Experimental Design - (a) Rat Behavioral Task with Four Holes. Each hole is associated with a given menu of concave/convex exposures to gains and losses; defining a profile (Anti Fragile, Robust, Vulnerable and Fragile) ; (b) Values and Ex-ante Frequencies for Convex (green)/Concave (red) Gains/Losses with the largest probabilities highlighted in blue (20-25

(a)

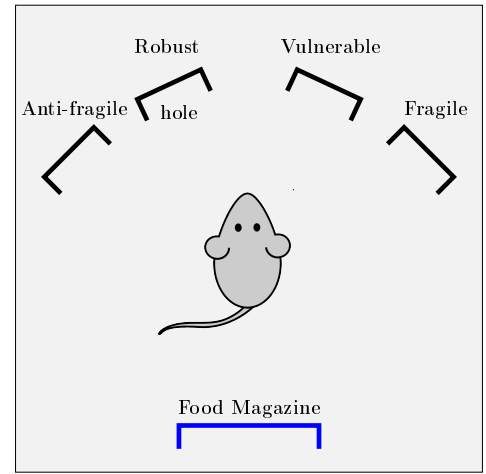

(c)
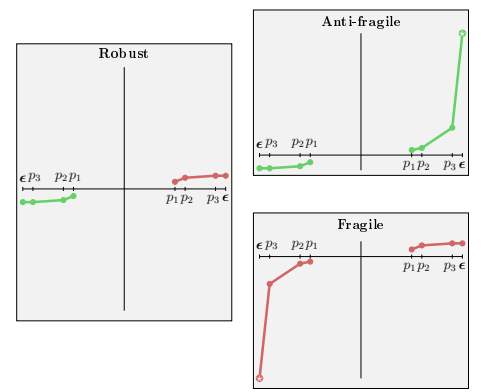

(b)

\begin{tabular}{|ccccc|}
\multicolumn{2}{c}{ Losses (sec) } & & Gains (pellets) \\
\hline 6 & concave & Probability & & concave \\
12 & 3 & $\approx 0.25$ & 1 & 2 \\
15 & 36 & $\approx 0.20$ & 3 & 4 \\
15 & 240 & $\approx 0.05$ & 12 & 5 \\
& & $\epsilon$ & 80 & 5
\end{tabular}

(d)

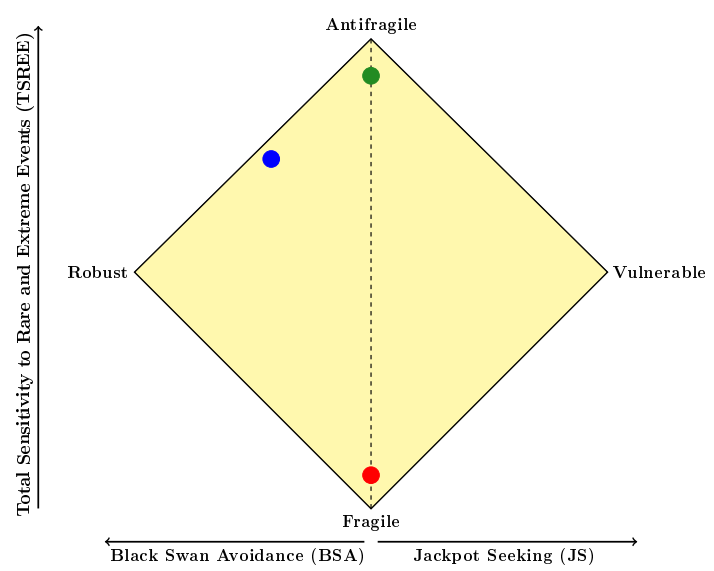

\section{Results}

\subsection{Design and Task}

In panel $(a)$ of Figure 1 is depicted a schematic representation of the Skinner box, in which all rats perform all experimental sessions. Each rat had the possibility at all times to poke into 4 holes, each corresponding to a different sequence of gains and losses, drawn at random for each rat and fixed accross sessions. Gains were sugar pellets while losses were time-out punishments (time period during which nosepoke remained inactive) measured in seconds (see Appendices A.1-A.2 for details). The key feature of the four possible options associated to each available hole was that they were combinations of convex (that is, accelerating) and/or concave (that is, decelerating) gains and losses, in panel (b) in Figure 1. While, as shown in the first and last columns, values for losses and gains differed for convex and concave options, the associated probabilities were assumed to be identical. They were set, as seen in the middle column, to $\left\{p_{1}=0.25-\varepsilon / 3, p_{2}=0.2-\varepsilon / 3, p_{3}=0.05-\varepsilon / 3, \varepsilon\right\}$ for both concave and convex gains. Parameter $\varepsilon$ is the (very small) ex-ante probability of the largest value that we label the Jackpot for the 80-pellet gain, and the Black Swan for the 240-second loss. Both events are denoted rare and extreme events (REE 
for short). ${ }^{2}$ The next largest values (that is, 12 pellets and 15 seconds, vs 5 pellets and 36 seconds) are labeled a rare event (RE), since their total probability is around $10 \%$ and the associated values are moderate compared to REE. Note that for concave gains and convex losses the RE and REE values coincide. This is to contrast with the Jackpot and Black Swan that occur only if convex gains and concave losses are chosen, respectively. With total probability about $90 \%$, therefore, the remaining set of lowest values in the first two rows (underlined in blue in panel $(b)$ of Figure 1) for concave and convex options are labeled normal events (NE). Obviously, combining convex/concave gains/losses delivers 4 possible options.

The 4 options that correspond to the 4 holes in the Skinner box are pictured in panel $(c)$ of Figure 1, with the following meaning. On the horizontal $x$-axis are reported the ex-ante probabilities that are unknown to the subject, which only observes the outcome that is measured on the vertical $y$-axis. ${ }^{3}$ For convenience, gains appear in the upper-right quadrant while losses appear in the lower-left one. For example, the option named Anti-fragile (after [18]) at the top of panel (c) is composed of convex gains and convex losses: the right part depicts the points in the $(x, y)$ axis are $\left\{p_{1}, 1\right\},\left\{p_{2}, 3\right\},\left\{p_{3}, 12\right\},\{\varepsilon, 80\}$, corresponding to the fourth column in panel $(b)$; the left part corresponds to the points $\{\varepsilon,-15\},\left\{p_{3},-15\right\},\left\{p_{2},-12\right\},\left\{p_{3},-6\right\}$, where "negative" values are interpreted as losses in seconds. Looking at the green curves that summarize the Antifragile option, then, one visualizes convexity directly from the properties that gains accelerate moving right from the origin, while losses decelerate moving left from the origin. As a consequence, the Anti-fragile option potentially delivers the Jackpot and certainly protects from the Black Swan. ${ }^{4}$

Symmetrically, the Fragile option in the lower part of panel $(c)$ in Figure 1 has both gains and losses that are concave (in red). Gains are $\left\{p_{1}, 2\right\},\left\{p_{2}, 4\right\},\left\{p_{3}, 5\right\},\{\varepsilon, 5\}$, which means that gains plateau at 5 pellets, compared with 80 for the Jackpot attached to convex gains. In the loss domain, the Fragile option delivers $\{\varepsilon,-240\},\left\{p_{3},-36\right\},\left\{p_{2},-9\right\},\left\{p_{3},-3\right\}$ : it exposes to the Black Swan with an awfully long delay of 240 seconds. For the Fragile choice, then, gains decelerate while losses accelerate, so that it potentially delivers the Black Swan but certainly avoids the Jackpot. Finally, the ways Robust and Vulnerable combine convex/concave gains and losses may be viewed as symmetric: the Robust choice protects from the Black Swan but at the same time misses the opportunity to get the jackpot, while the Vulnerable option potentially delivers both REE since both gains and losses accelerate!

In sum, the four options in panel $(c)$ of Figure 1 are therefore combinations of the convex/concave gains/losses in panel (b): the Anti-fragile option at the top is convex both in gains and in losses, while the Robust option (left) is convex only in the loss domain. On the other hand, the Vulnerable exposure (right) has convex gains only, while the Fragile option (bottom) is concave both in gains and in losses. This implies that the Jackpot of 80 pellets may materialize only when either the Anti-fragile or the Vulnerable exposure are picked, while the Black Swan of 240 seconds may be experienced only by rats choosing either the Fragile or the Vulnerable exposures. In Appendix A.3 it is shown that convex gains/losses dominate concave gains/losses in the sense of Stochastic Dominance, provided that the probability of REE $\varepsilon$ is larger

\footnotetext{
${ }^{2}$ As a convenient shortcut, therefore, the Black Swan is thought of as a rare and extreme loss, to differentiate it from a rare and extreme gain.

${ }^{3}$ In contrast with [4], we do not give rats during the training sessions any cue about the events' frequencies, which are set by us as experimenters. This makes our setting ecologically closer to experience-based designs.

${ }^{4}$ Although in panel $(c)$ convex (concave) gains and losses appear in green (red), a geometric description might be useful as well. For example the property that a monotone, upward or downward, convex (concave) curve lies below (above) its chords joining any pair of points can be used to identify green (red) curves.
} 
than about $0.3 \%$. In our design, the values for $\varepsilon$ we assume meet this condition but still are below $1 \%$, which admittedly ensures that REE are indeed rare.

Panel $(d)$ in Figure 1 is the central graphic tool for presenting the main data that result from our experiments. Because, as emphasized so far, convexity of available options is a key dimension in our design about decision-making under REE, our first task is to track how often rats have chosen convex rather than concave options in gains/losses, over the course of the 40 sessions that each of our 20 rats has run. Second, we also aim at assessing whether rats have possibly picked convex options more often in the loss domain than in the gain domain, that is, in an asymmetric fashion. Both dimensions easily combine in panel $(d)$. On the vertical $y$-axis we report the sum of the frequencies of choosing both convex gains and convex losses, while on the horizontal $x$-axis is depicted the difference, defined as the frequency of picking convex options in the gain domain minus the frequency of choosing convex options in the loss domain. The resulting rotated square in panel $(d)$ of Figure 1 is then a convenient way to report each rat' choices over one or several sessions, along both dimensions and therefore to group rats.

To see this, let us first focus on the 4 edges in panel $(d)$, which represent extreme cases in the sense that they correspond to exclusive choices: if an hypothetical rat's behavior is summarized by a point located at exactly the Anti-fragile edge, it means that it rat has chosen the Anti-fragile option all the time. As a consequence, the vertical $y$ coordinate reaches its maximum value (say, 2 if frequencies are added up) while the horizontal $x$ coordinate is zero since both convex gains and convex losses are symmetrically chosen all the time. Following the same logic, the Fragile edge has coordinate $\{0,0\}$ since it involves choosing concave gains and losses all the time, hence never convex options. The Robust option, in contrast, is depicted by $\{-1,1\}$ since it implies picking exclusively convex losses but concave gains, in an asymmetrical fashion. The reverse asymmetry characterizes the Vulnerable edge at $\{1,1\}$, with convex gains and concave losses chosen at all times. Now when a rat's behavior happens to be represented by a point that is not located at any of the 4 edges, such a location indicates that that rat diversify across options. For example, the green point in panel $(d)$ is close to the Anti-fragile edge and located on the vertical line linking it to the Fragile edge, which might happen if a rat would pick the former with frequency about $95 \%$ of the time and the latter about $5 \%$ of the time. The red point would be attained by reversing those proportions. Of course many other combinations of the 4 options is possible. For example, the blue point depicts a hypothetical rat that would diversify across the Anti-fragile and Robust options almost equally, at the expense of the two remaining options that have negligible frequencies. Next section will show that diversifying primarily across two options is the main pattern featured in our behavioral data.

A feature of our experimental design and its implications need be emphasized in more details at this point. First, the set of NE has total probability of about $p_{1}+p_{2} \approx 0.9$ since $\varepsilon$ is a small number. RE are rare but not extreme is the sense that their total probability $p_{3} \approx 0.1$ is moderately small while their consequences are moderately large. REE, in contrast, are more extreme outcomes, since they imply waiting 240 seconds (the Black Swan) and gaining 80 pellets (the Jackpot), with a much smaller likelihood. In practical terms, the frequency of each REE is ex-ante around $1 \%$ for all rats. Despite their small probability, REE have some importance in the following sense. As shown in Appendix A.3, the values for both frequencies and outcomes ensure that concave options dominate, in the sense of first-order stochastic dominance, convex options over NE, as well as in the sense of second-order stochastic dominance over the union of NE and RE. In other words, if the extreme gains and losses do not materialize, concave gains (losses) dominate convex 
gains (losses). However, this does not hold when REE are added: provided that $\varepsilon \leq 0.302 \%$ (which we exante assume), convex gains (losses) dominate concave gains (losses) in the sense of second-order stochastic dominance. This implies, in particular, that over the full domain that is the union of NE, RE and REE, expected gains for convex options are larger than expected gains for concave options. Likewise, expected time-out punishments for convex options are smaller than expected gains for concave options.

The reason that we impose such a dominance reversal is as follows. In theory, a rat choosing concave options at all times can be thought of as ignoring, i.e. as having absolutely no sensitivity to, rare and extreme events: it acts as if those events never occur and always go for first or second-order stochastic dominance (over NE and RE) as decision-making criteria. And those criteria unequivocally point to the Fragile option as the best choice. This gives our first measure along the $y$-axis of panel $(d)$, Total Sensitivity to Rare and Extreme Events (Total Sensitivity for short), that again simply sums up the proportion of convex exposures that are chosen for each rat over the 40 sessions. A rat picking the Fragile option at all times has zero Total Sensitivity, since it behaves as if the Black Swan or the Jackpots never occur. At the other extreme, a rat picking exclusively the Anti-fragile option signals maximal Total Sensitivity, since it always stays exposed to the Jackpot while always avoiding the Black Swan. Moderate Total Sensitivity arises, then, if a rat diversifies its choices, for instance primarily across either Anti-fragile and Fragile, or Robust and Vulnerable. Note that such pairs obviously achieve identical outcomes in terms of pellets and time-out punishments.

Because gains and losses are deliberately integrated in our design, we also need to distinguish whether rats tend to choose convex exposures symmetrically over the gain and loss domains. This is the purpose of the measure along the $x$-axis in panel $(d)$. We say that a particular rat exhibits Black Swan Avoidance when it picks convex exposures in the loss domain more often than in the gain domain. Symmetrically, Jackpot Seeking is the label we use to depict a situation when convex choices are more often made in the gain domain. Given its Total Sensitivity, a rat's behavior exhibits Black Swan Avoidance (Jackpot Seeking) whenever it is represented by a point that is located on the right (left) half of the square in panel $(d)$. In other words, the numbers read on the $y$-axis measure One-Sided Sensitivity to Rare and Extreme Events (One-Sides Sensitivity for short): when negative (positive) it indicates Black Swan Avoidance (Jackpot Seeking).

\subsection{Total and One-sided Sensitivity to Rare and Extreme Events}

In this section, we present the behavioral data coming from the 40 sessions that we have been running for each of the 20 rats. We organize the data in the following way, so as to underline two features of the choices made by the sample of rats. In each panel of Figure 2 is depicted the (rotated) square that we have already presented in panel $(d)$ of Figure 1, the edges of which represent the four exposures if chosen exclusively at all times. In the square, each point represents a rat. A quick glance at Figure 2 already shows that virtually no such point is located exactly at any of the 4 edges. Quite the contrary and strikingly, it turns out that all rats diversify their choices across a set of options, which is reminiscent of observed behaviors such as, for example, bet-hedging in animals and financial portfolio strategies used by humans (see [2], [14], [16], [17]).

How rats diversify their choices can be represented in three steps, focusing first on all choices made by all 20 rats over the 40 sessions, depicted by black points in panel $(a)$ of Figure 2. More precisely, each 
Figure 2: Total Sensitivity to REE ( $y$-axis) against One-sided Sensitivity to REE ( $x$-axis; i.e. REE seeking towards the right and avoiding towards the left) - (a) black-colored points depict 40 sessions run by 20 rats all together; (b) in each square representing a rat, colored points depict 40 sessions for each of the 20 rats; the color code indicates the 2 main categories of profiles observed (blue: the most sensitive to REE and black swan avoiders; green: sensitive to REE but neither biased towards seeking nor avoidance (close to midline on the graph) and 2 outliers were identified and color-coded in red and pink. (c) color-coded points depict averages over the 40 sessions for each of the 20 rats

(a) All sessions

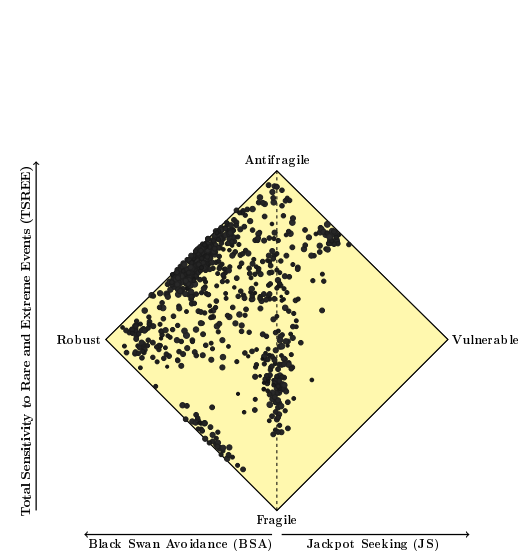

(b) By rat

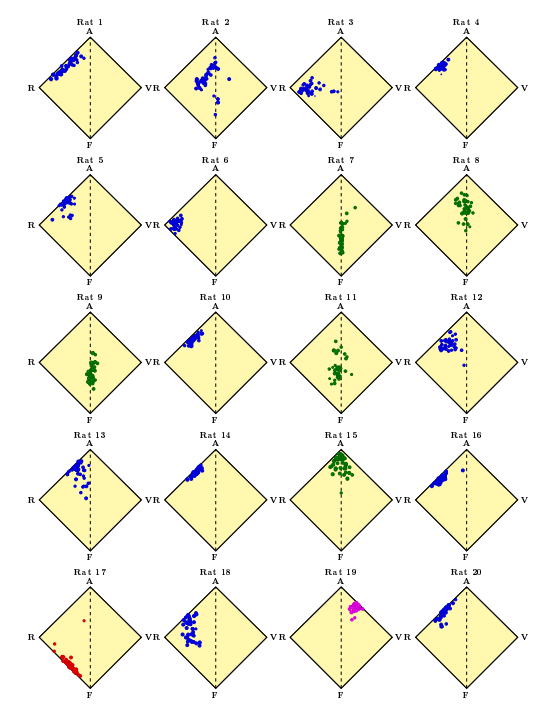

(c) Mean behavior

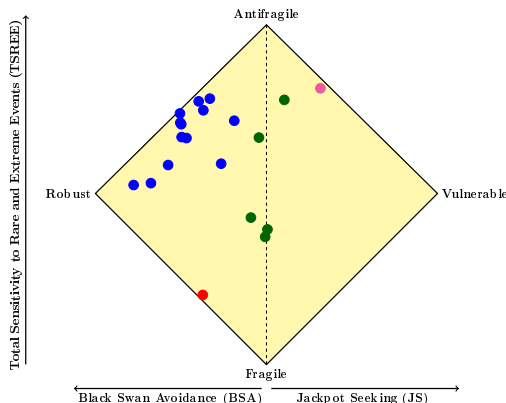

black represents a rat's session, with a total of 800 such points. It is clear that most points lie on the right half of the square, that is, most points lie on the Black Swan Avoidance side. In addition, within the right half most points are located in the upper fourth, that is, above the Robust-Vulnerable vertex, suggesting moderate to high Total Sensitivity. These two patterns are better seen if we focus on each individual rat.

We now look separately at the individual behavior for each of the 20 rats across the 40 sessions, in panel (b) of Figure 2. Each rat is represented by a square and, within each square, a point represents one of the 40 sessions for that particular rat. For convenience, the vertices of all squares are labelled by letters: "A" obviously stands for the Anti-fragile exposure, while "R", "V" and "F" stand respectively for the Robust, Vulnerable and Fragile exposures. All rats exhibit moderate to high Total Sensitivity, with the exception of rat 17 depicted in red in panel $(b)$ of Figure 2, which is an outlier. Among this vastly dominant group, one can identify two sub-groups though, color-coded in blue and green, and a second outlier. Starting with the latter, rat 19 (in purple) is the sole rat that exhibits both the highest Total Sensitivity and Jackpot Seeking, by mixing mostly Anti-fragile (more often) and Vulnerable (less often) options. ${ }^{5}$

Except for these two very different outliers (rats 17 and 19), the remaining 18 rats can be gathered in two sub-groups. The largest one gathers the rats colored in blue: rats 1 to $6,10,12$ to $14,16,18$ and 20 diversify their choices primarily across the Robust and Anti-Fragile options, with various frequencies. This

\footnotetext{
${ }^{5}$ Footnote here on this interesting outlier in terms of addictive behavior?
} 
means that the dominant group, composed of 13 rats depicted in blue, exhibits both moderate to high Total Sensitivity together with Black Swan Avoidance: rats within this group more often try to avoid the Black Swan that to be exposed to the Jackpot. A smaller sub-group composed of the rats depicted in green, however, rather diversify their choices across the Fragile and Anti-Fragile options: those 5 rats depicted in green exhibit also moderate to high Total Sensitivity together with Black Swan Avoidance but feature neither Black Swan Avoidance nor Jackpot Seeking: those rats have zero One-Sided Sensitivity or, in other terms, make choices that feature symmetry towards avoiding the Black Swan and seeking the Jackpot.

Third those two groups are conveniently represented in panel $(c)$ of Figure 2. Each point is associated to a particular rat and it represents the average of its choices over the 40 sessions, as measured through our two metrics. As for Total Sensitivity, one notices that all rats but 4 have an average sensitivity larger than half its maximal value: 3 rats having a Total Sensitivity slightly below 1 and a single rat with a value around 0.5. The latter (rat 17 in red) is the closest to a Fragile behavior, with a significant deviation from it, though. Overall, therefore, most rats (that is, 19 out of 20) are rather sensitive to the presence of rare and extreme events. In addition, panel $(c)$ confirms the striking feature that most rats exhibit Black Swan Avoidance, that is, negative One-Sided Sensitivity. Rats 17 and 19 (in red and purple respectively) are outliers for opposite reasons. The latter is the unique rat with low Total Sensitivity while the latter is the rat with the largest Total Sensitivity and Jackpot Sensitivity. Among the remaining group composed of 18 rats, all with moderate to high Total Sensitivity, the average behaviors in panel $(c)$ allow one to identify the two sub-groups that we have previously stressed: the 5 rats depicted in green have zero One-Sided Sensitivity (neither Black Swan Avoidance nor Jackpot Seeking) while the 13 blue rats exhibit moderate to high Black Swan Avoidance.

An interesting characteristic appears from direct inspection of panel $(c)$ in Figure 2: the sample of 16 rats with the largest Total Sensitivity features a positive relationship between Total and One-Sided Sensitivity. In other words, among those rats that have both moderate to high Total Sensitivity and Black Swan Avoidance, rats that are more sensitive overall to convexity tend to also exhibit lower Black Swan Avoidance (that is, less negative One-Sided Sensitivity). It is as if large Total Sensitivity comes with a mixture of exposures that tend to favor convexity in the gain domain relative to the loss domain. In a sense, rat 19 in purple can be seen as a limiting example of that behavior: it combines the Anti-fragile and Vulnerable options so as to approach near-maximal Total Sensitivity and Jackpot Seeking as opposed to Black Swan Avoidance.

This property is confirmed if we compute the correlation between average sensitivity and average asymmetry (Appendix A.3). For example, for the whole sample of 20 rats, this correlation is close to (and not statistically different from) zero, while it has a value of about 0.57 over the 40 sessions within the group of 16 rats with Total Sensitivity larger than one. In other words, if we drop the 4 rats with the smallest Total Sensitivity from the sample (those rats represented by points below the Robust-Vulnerable vertex in panel (c) in Figure 2), we are left with 16 rats for which larger Total Sensitivity is associated with lower One-Sided Sensitivity/less Black Swan Avoidance, that is, more symmetry and even Jackpot Seeking for rat 19. Moreover, if we divide the 40 sessions of the 16-rat group over time, the correlation between Total and One-Sided Sensitivity increases from about 0.17 on average in the first 10 sessions, to 0.56 in the next 10 sessions, to 0.71 in the next 10 sessions, and finally to 0.64 in the final set of 10 sessions. Therefore, the 
Figure 3: Responses of Total Sensitivity to REE in panel (a), and of One-sided Sensitivity to REE in panel (b), to Jackpots (blue) and Black Swans (red) for each of the 20 rats. The black line materialize no change in sensitivity after exposure to the REE compared to before.

\section{(a) TSREE}

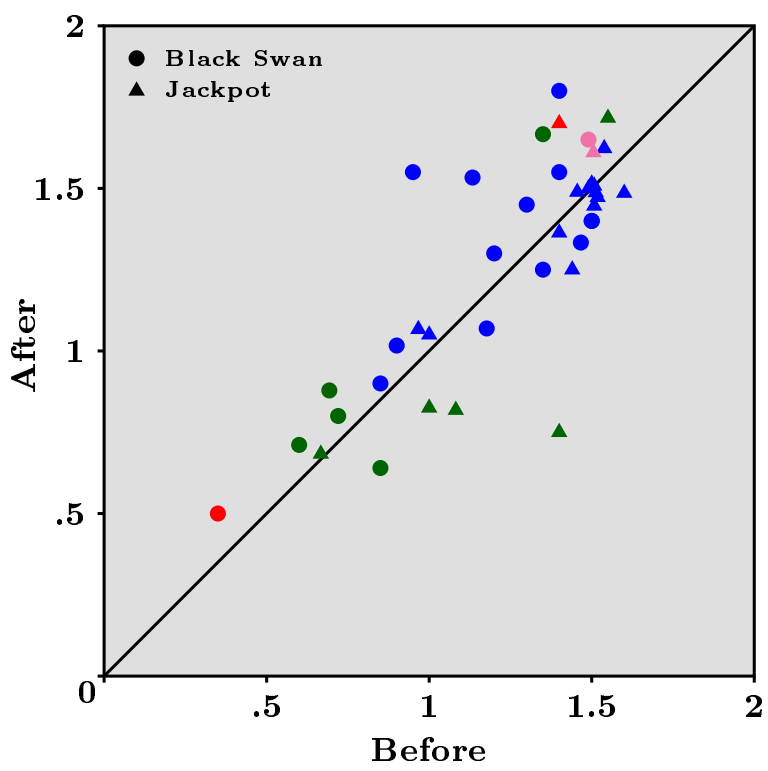

(b) BSA \& JS

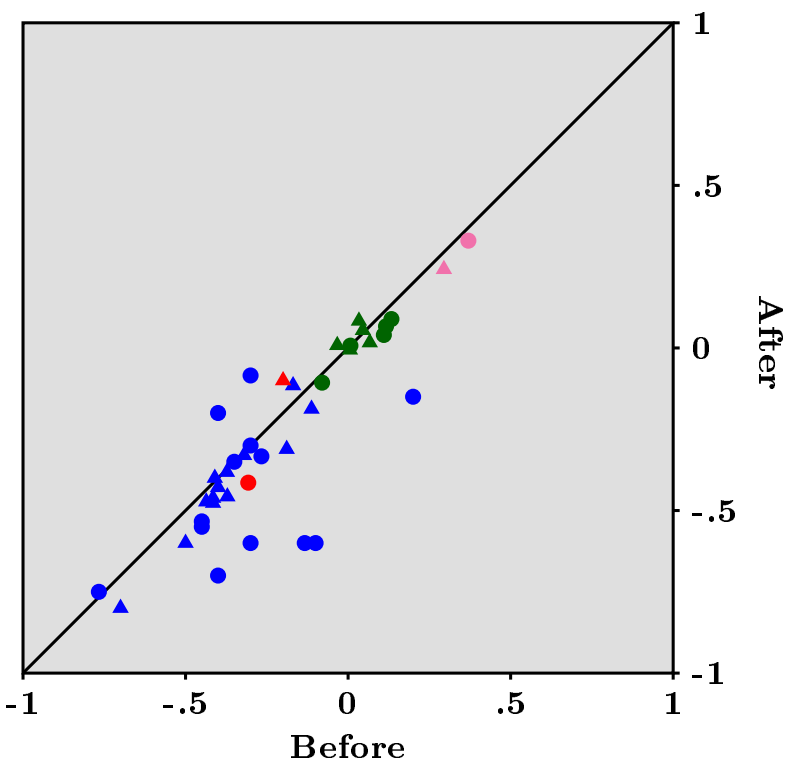

pattern we alluded to appears early in the sessions and is consistent over time.

\subsection{Response to Rare and Extreme Events}

Having documented that most rats (19 out of 20) exhibit medium to high Total Sensitivity and medium to high Black Swan Avoidance, we now address the issue of how rats respond to the actual occurrence of a rare and extreme event within a session. More precisely, we aim at comparing the average behavior of each rat over the 10 nose pokes that precede extreme events, together with the 10 nose pokes that follow the occurrence of REE, either a Black Swan or a Jackpot. In Figure 3, we report how REE affect Total and One-Sided Sensitivity, within a window of 10 nose pokes before and 10 nose pokes after. For both panels $(a)$ and $(b)$, each point represents a particular rat and the colors group all rats as in panels $(b)-(c)$ of Figure 2). The new information is captured by dots representing Black Swans and triangles representing Jackpots, the occurrence of which delineates the before and after.

Visual inspection of Panel $(a)$ in Figure 3 suggests we that while Jackpots tend to not affect Total Sensitivity much, Black Swans do and tend to increase it: most dots do tend to be located above the 45-degree line, while triangle do not. In other words, extreme losses/Black Swans tend to reinforce Total Sensitivity, contrary to extreme gains/Jackpots. This reinforcement effect due to experiencing negative REE is consistent with our main result that most rats are sensitive and averse to extreme losses, that is, exhibit medium to high Total Sensitivity and Black Swan Avoidance. Panel (b) of Figure 3, in addition, reveals that extreme losses tend to make Black Swan Avoidance stronger: most dots tend to be located 
below the 45-degree line, while triangles are more or less aligned on this line. Again, this is consistent with the observation that extreme losses are somewhat given more weights than extreme gains by most rats, which implies that Black Swan Avoidance is reinforced when a extreme loss is experienced. Such an eye-ball analysis is confirmed by statistical test, including one using bootstrap, as shown in Appendix A.3. In sum, a within-session analysis of the effects of experiencing a REE shows that the occurrence of Black Swans tends, in the short-run, to significantly increase both Total Sensitivity and Black Swan Avoidance, while the occurrence of Jackpots tend to leave Total and One-Sided Sensitivity unaltered.

So we mentioned adding a between-session analysis of the effects of REE here: are they persistent over the long-run, that is, over sessions?

\section{Discussion}

\section{$3.1 \quad$ Outcomes}

What are the ex-post outcomes of the choices made by the different rats over the course of all sessions? In the first left columns of Table 4 in Appendix A.3, we report for each rat the number of nose pokes, the number of REE of each type the rat has experienced. We also report, for the rewards in pellets and the waiting times in seconds, the sum as well as the first four moments of the outcome per nose poke. The overall pattern that emerges from Table 4 is that a typical rats in with high Total Sensitivity group tends to have outcomes that differ from a typical rat with moderate Total Sensitivity. For gains (i.e. sugar pellets), the former's rewards have typically higher mean and variance but smaller skewness and kurtosis that the later's. This happens for instance, to an extreme degree, when we compare rat 15 (the most Anti-fragile rat) and rat 17 . On the loss side, by symmetry the waiting time of the average high-sensitivity rat tends to have lower mean and variance, but larger skewness and kurtosis. These facts are consistent with the fact that a typical high-sensitivity rat tend to pick a mix of exposures that is more convex both on the gain domain and on the loss domain, compared to an average low-sensitivity rat.

\subsection{Convexity Premiums}

Evidently the outcomes that we report in Table 4 depend on the convexity mix of options that each rat has chosen, as captured by our notions of Total and One-Sided Sensitivity. To go beyond Table 4 so as to capture the extent to which rats exploit convexity in their choices, it is perhaps informative to look at the outcomes for each rat in the following way. Right and left panels in Figure 4 refer to losses and rewards, respectively. In each panel, rats are color-coded as in the previous figures, and they are represented horizontally by two segments which are normalized in the following way. Consider for example (blue) rat 1 in the first line. In the right panel about pellets, the point of the black segment most to the left represents the outcome that would have happened, had the rat chosen a concave option in the gain domain (that is, either Robust or Fragile), exclusively in all sessions. The point most to right corresponds, in contrast, to the counterfactual outcome in which all nose pokes of the rat correspond to a convex option ((that is, either Vulnerable or Anti-Fragile). Superimposed on this background black segment, is a bold-colored segment 
Figure 4: Convexity Premiums for each of the 20 rats (row)

Each line represents a rat and the color dot indicates to which extend the animal has benefited

from their choices. Towards the right, the dot materializes how many pellets were obtained relative to the number that would have been obtained if a convex menu had been chosen at each trial. Towards the left, the coloured dot indicates the total seconds of penalty obtained relative to what would have been obtained if a concave menu had been chosen at each trial. The color code indicates the profile type of the animal, as in the previous figures (see fig 2).

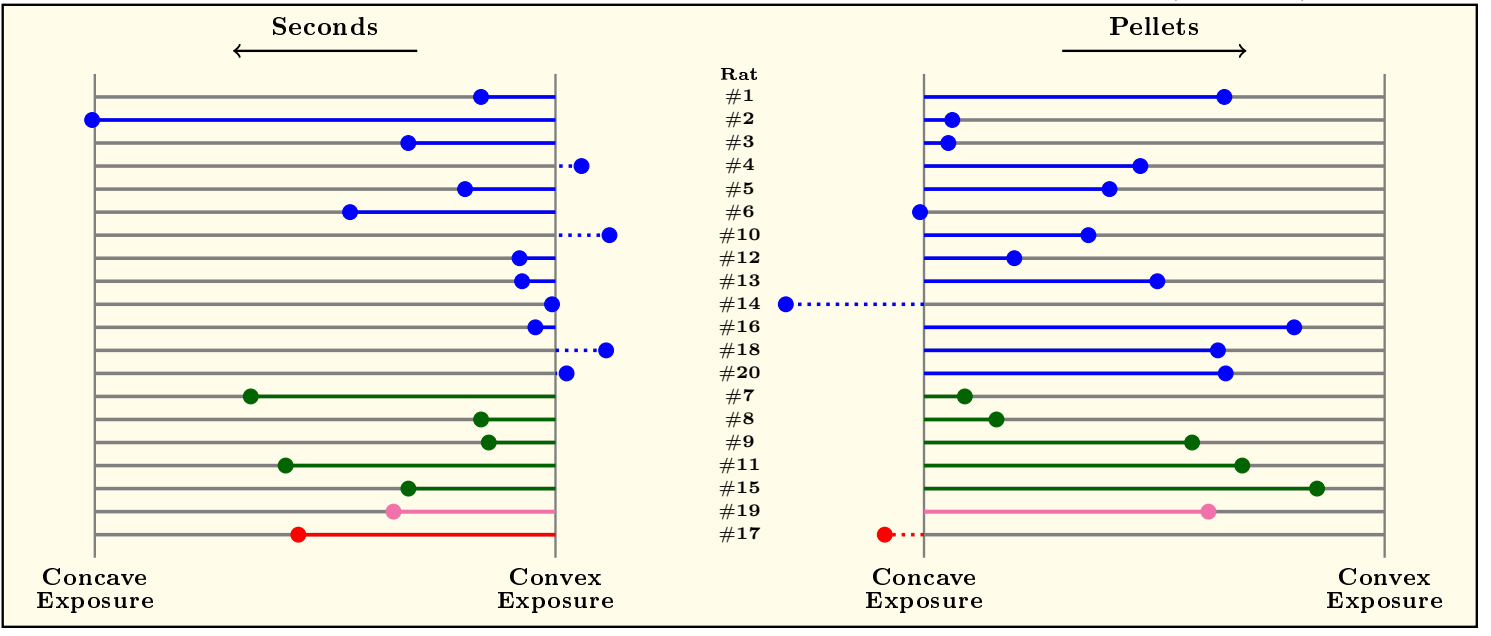

(blue because rat 1 is blue) that ends with a circle indicates what the rat has gained due to convexity in relative terms - what we call the convexity premium. Rat 1 has a normalized convexity premium that corresponds to roughly $80 \%$ of what it would have got, in terms of sugar pellets, had he counterfactually chosen to mix only Vulnerable and Fragile, instead of Robust and Anti-fragile as it did. Similarly, one sees from the right panel of Figure 4 that the Anti-fragile rat 15 has a convexity premium that corresponds to roughly $90 \%$ of what it would have got, in terms of sugar pellets, had he chosen to mix only Vulnerable and Fragile. From panel (b) of Figure 2, we know that rat 15 has also, more rarely, picked the Robust and Fragile exposures and this is why its convexity premium in the gain domain is less than maximal. At the other extreme, the rat 6 has a convexity premium near zero. Note that two rats our outside the black segment in the gain domain, that is, have "negative" convexity premiums, and they are indicated by dashed lines. Rat 17 has picked the Anti-fragile exposure a few times but got only one extreme gain, while rat 14 is more sensitive and hence got more extreme gains but still too few of them. Therefore, both rats got less sugar pellets than what they would have gotten by mixing exposures that are concave in the gain domain (Robust and Fragile).

The left panel in Figure 4 about time-out punishments can be read in a similar way, with now the point most to the left of the black segment corresponds to the largest loss in terms of waiting times, while the end point to the right corresponds to the lowest amount of time wasted. Therefore, the bold and colored segment indicates a negative premium: for instance, rat 2 on the first line in the left panel of Figure 4 has been exposed to the largest time-out punishment: it has a negative and large convexity premium because it mixed exposures with concave losses (that, is Fragile and Vulnerable), too often. Symmetrically, some rats turn out to get convexity premiums that are above $100 \%$ because they got too few black swans when they 
picked exposures that are concave in the loss domain). Comparing the right and left panels in Figure 4, one infers that rats exploit convexity better in the loss domain than in the gain domain, that is, they more often avoid Black Swans than they get Jackpots. This is of course consistent with our earlier observation that most rats exhibit moderate to high Black Swan Avoidance.

\subsection{Limitations}

The focus of our experimental design is on rare and extreme events under radical uncertainty connects our study to the sizeable literature which contrasts experience-based to description-based settings. Although such a distinction might seem to apply to human subjects only, it is possible to give animals some cues that are related to probabilities (see e.g. [4]). In our experience-based setting, there is sense in which our results somehow qualify the phenomenon of under-weighting of rare events that is typically found in the literature (see for example [7]): most rats in our experiments exhibit significant sensitivity to REE in the form of moderate to high TSREE and BSA.

In addition, some limitations of our analysis are worth mentioning, as they constitute avenues for further research. First, one main result of this study is that most rats tend to mix 2 out the 4 available options, as opposed to exploiting one option almost or exploring all options almost exclusively and consistently over time. This fact only suggests that part of our results might be related to spontaneous alternation behavior, that is well documented for rats and other animals (see e.g. [5]) and which one might want to control for when measuring the extent of diversification in the context of options with REE. Second, there is substantial heterogeneity among rats in terms of choice over all four options. This is an interesting starting point to examine the extent with which social interaction, such as dominance and cooperative behaviors for example, might affect animals' choices in similar context. This is especially important since rare and extreme events might lead to extinction of groups or species. Finally, although the result reported in this paper relate to experiments in which subjects are rats, the proposed design can be, and in fact has been adapted to humans and other animals. In ongoing research, we have run pilot experiments to inquire into whether TSREE is also observed among human subjects. We believe that results from similar experiments among different species might be of interest for the analysis of neurobiological substrates involved in decision-making and its evolutionary traits in the context of rare and extreme events.

\section{Conclusion}

In this paper we present a novel experimental design that aims at measuring the extent to which animal subjects are sensitive to rare and extreme events and, in addition, how rats respond to those very unfrequent events under radical uncertainty. At the heart of such a design is the notion that convex options are attractive because they allow to gain from uncertainty in the presence of rare and extreme events, in terms of both gaining sugar pellets and avoiding time-out punishments. Although the task itself can be thought as a traditional four-armed bandit task, with four options to choose from at any time, the novelty of our design is that it provides two direct measures that help interpreting our behavioral data: Total Sensitivity to Rare and Extreme Events, and One-sided Sensitivity to Rare and Extreme Events with Black-SwanAvoidance/Jackpot-Seeking behaviors as limiting cases. Using our setup on a sample of 20 rats, with about 
6000 stimuli per rat, we document two sets of results. First, most rats (19 out of 20) can be grouped into a moderate to high Total Sensitivity group. This means that most rats diversify their choices across options in such a way that they more often rely on convex ones than on concave ones overall. Therefore, they tend to seek extreme gains/Jackpots and to avoid extreme losses/Black Swans. In addition, most rats (15 out of 20) tend to exhibit Black Swan Avoidance, which indicates that, given Total Sensitivity, they tend to try more often to avoid Black Swans than to seek Jackpots. We interpret such a behavior as significant aversion towards uncertainty about rare and extreme losses. Consistent with such an interpretation, both ex-post outcomes and counterfactual analysis using convexity premiums, for each rat over all sessions, together show that our sample of animal subjects does a better job at exploiting convexity in the loss domain than in the gain domain.

\section{A Star*Methods}

\section{A.1 Experimental Model and Subject Details}

\section{Animal Subjects}

Adult Lister Hooded males ( $\mathrm{n}=20,200 \mathrm{~g}$ at arrival, Charles River) were housed in groups of two in Plexiglas cages and maintained on an inverted $12 \mathrm{~h}$ light/dark cycle (light onset at $7 \mathrm{pm}$ ) with water available $a d$ libitum, in a temperature - and humidity - controlled environment. Food was slightly restricted ( $80 \%$ of daily intake). Animal care and use conformed to the French regulation (Decree 2010-118) and were approved by local ethic committee and the University of Aix-Marseille under \#03129.01.

\section{A.2 Method Details}

\section{Apparatus}

All behavioral experiments took place during the animals' dark phase in standard five-hole operant boxes (MedAssociates) located in ventilated sound-attenuating cubicles. One side of each box was equipped with a central house light, a tone generator and a food magazine, outfitted an infrared beam for detecting nose poke inputs. Sucrose pellets (20 mg; Bio-Concept Scientific) were delivered from an external food pellet dispenser. An array of five response holes was located on the opposite wall, each equipped with stimulus lights and infrared beams for detecting input. The center hole was continuously closed throughout the experiments (Fig. 1a). Data were acquired on a PC running MedPC-IV.

\section{Design of Behavioral Sequences}

Menus were elaborated by mixing convex and concave exposures for both the gain (sugar pellets) and loss (time-out punishment) domains. Each exposure included four different events (Figure 1 panel $(b)$ ). In the gain domain, animals could obtain 1, 3, 12 or 80 sugar pellets in the convex exposure or 2, 4, 5 or 5 pellets with concave exposure. In the loss domain, convex exposure may impose 6, 12, 15 or 15 sec of time-out punishment or $3,9,36$ or $240 \mathrm{sec}$ for the concave exposure.

The four behavioral options depicted in Figure 1, panel $(c)$, are therefore combinations of the above convex and concave exposures: the Anti-fragile exposure at the top is convex in both domains, while the Robust option (left) is only convex in the loss domain. On the other hand, the Vulnerable exposure (right) is convex 
only in the gain domain, while the Fragile option (bottom) is all concave. This implies that the extreme but rare - gain of 80 pellets (thereafter termed jackpot) may be delivered only when either the Anti-fragile or the Vulnerable options are picked, while the extreme and rare time-out punishment of 240 sec (thereafter termed black-swan) may be only experienced by choosing either the Fragile or the Vulnerable options.

The first three events of each behavioral options belong to the frequent domain as their frequency of occurrence, respectively $0.5,0.4$ or 0.1 , is significantly larger than zero. During behavioral testing, animals equivalently experienced the gain and loss domains. On the other hand, extreme outcomes, i.e. Jackpot or Black Swan, have a much smaller likelihood of occurring since they may appear only at particular point during the behavioral sequences (see below). This means that they could happen if a rat has chosen an exposure that is either convex in the gain domain or concave in the loss domain at a given time. This implies that the frequency of rare and extreme events is less than half of one percent for all rats. Despite their low probability, extreme events have some importance in the following sense. Our calibration of both frequencies and outcomes ensures that concave exposures dominate convex exposures, in the sense of ex-post first order stochastic dominance, over the frequent domain. In other words, if the extreme gain does not materialize, the expected payoff in sugar pellets is larger for concave exposures than convex exposures. Similarly, the expected time-out punishment is lower for concave exposures compared to convex ones, if the black-swan does not happen. However, ex-post first-order stochastic dominance is reversed in the presence of extreme events, in which case convex exposures dominate concave ones. The reason we imposed such a dominance reversal was as follows. In theory, subjects always choosing concave exposures could be thought of as having absolutely no sensitivity to rare and extreme events, in the sense that they act as if those events never occur and always go for first-order stochastic dominance. This gives us our first measure, sensitivity to rare and extreme events (SREE for short), that simply sums up the proportion of convex exposures that are chosen for each rat over the 40 sessions. Because we deliberately integrate gains and losses in our design, we need to distinguish whether rats tend to choose convex exposures symmetrically over the gain and loss domains. We say that a particular rat exhibits Black Swan Avoidance (BSA for short) when it picks convex exposures in the loss domain more often than in the gain domain. Likewise, Jackpot Seeking (JS for short) obtains when convex choices are more frequent in the gain domain.

To avoid potential learning of the event occurrence during behavioral training and testing, ten different sequences of events, with respect of the first-order stochastic dominance as well as the balance between gain and loss domain exposures, were generated and randomly used for behavioral training and testing (Supplemental Figure 6). Furthermore, to increase the rarity and the unpredictable nature of extreme events, the sequences of events used during behavioral training and testing were declined in seven sequencetypes, in which jackpot and black-swan are either unavailable, solely or both available at a given time point of the sequence of events (Supplemental Table 1): when available, extreme events could be obtained at the 10th or the 60th activation within a given sequence-type, but could not occur at the same time. For example, in a behavioral sequence-type where the jackpot is available at the 10th position, any poke in the Anti-fragile and Vulnerable holes following nine activations in one of these menus (regardless of the positive or negative outcomes) would trigger the delivery of the jackpot. Of note, depending on the sequence-type used, animals would experience both extreme events in a single session.

\section{Behavioral Training and Testing}

Training was divided into five distinct phases before the final test: acquisition of the food collecting responses, 
acquisition of nose poking in the holes, training with four holes, attribution of menus to hole and training on the menus (Figure 5). Each session started with the illumination of the house light.

1. Acquisition of the food collecting responses: Animals were trained to collect sucrose pellets in the food magazine during three 30-min daily sessions (100 pellets max) under fixed ratio 1 schedule of reinforcement (FR1): one nose poke into the food magazine triggered the delivery of one sucrose pellet. During this initial phase, activation of other holes had no programmed consequences.

2. Acquisition of nose poking in the holes: Here, animals had only access to one hole (the three others being closed) during the 20-min sessions. One nose poke in the open hole triggered the illumination of the hole light and the delivery of one sucrose pellet in the food magazine. Perseverative pokes (those performed before food collection) had no consequences. Following food collection in the food tray, animals were allowed to poke again in the opened hole. During each session, a maximum of 100 pellets were delivered. All animals were trained twice on each hole.

3. Training with four holes: Following the eight training sessions, animals were allowed to poke in the four different holes during twenty daily sessions of 20-min. Nose poke in any hole triggered the illumination of the associated light and the delivery of one sugar pellet in the food magazine. Both perseverative activations and pokes in other holes had no consequences. After food collection in the magazine, animals were allowed to nose poke again in any hole. The first ten training sessions were limited to 100 sugar pellets. During the following ten sessions, animals were able to collect up to 200 pellets per session.

4. Attribution of menus to hole: We determined the spatial preference for each rat by establishing the percentage of activation of each hole during the last ten sessions. To favor the emergence of Anti-fragile choices, menus' attribution was made as follow:

- Anti-fragile exposure was associated to the preferred hole

- Robust exposure was associated to the 2 nd preferred hole

- Vulnerable exposure was associated to the 3rd preferred hole

- Fragile exposure was associated to the least preferred hole

5. Training on the exposures: Here, animals were first trained on the gain domain, i.e. no time-out punishment, for each menu/hole. They were subjected to two 20-min sessions, with unlimited number of pellets, during which only one hole was available (eight training sessions in total). For Anti-fragile and Vulnerable options, we used the two sequence-types where the jackpot was available at the 10th and 60th activations (Supplemental Table 1) to ensure that all individuals could experience both an early and delayed jackpot during training. Animals were then allowed to explore all gain options (four opened holes) during 9 20-min sessions, for which different behavioral sequence-types were used. Before training on the loss domain of the different menus, animals were first exposed to a mild and constant 3-sec time-out punishment. Here, animals had access to all options, but half of the activations lead to a 3-sec time-out punishment, notified by a 3-sec tone and the extinction of the house light. During this period, pokes in the different holes or the food magazine had no programmed consequences. After the 3-sec time-out punishment, the house light was turned on and the animals could again pokes in the different holes. Following nine 20-min training sessions, the loss domain of 


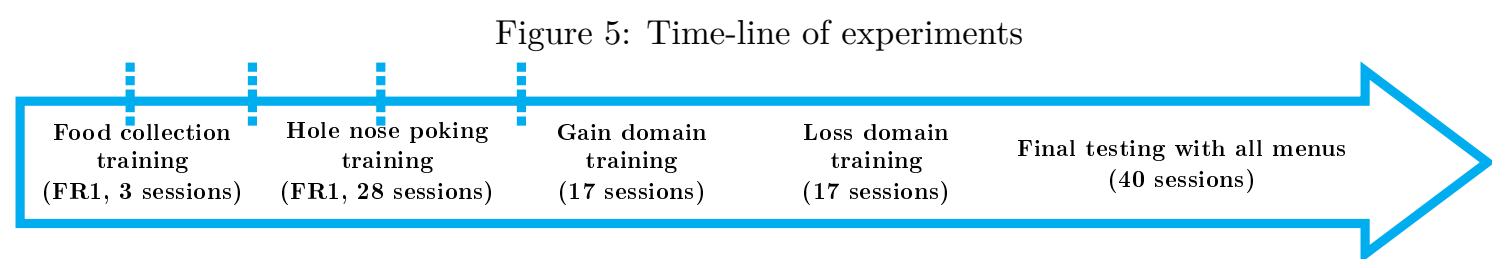

Table 1: Position of extreme event in the sequence

\begin{tabular}{ccc}
$\#$ & Jackpot & Black-Swan \\
1 & & \\
2 & 10 & \\
3 & 60 & \\
4 & & 10 \\
\hline 5 & & 60 \\
\hline 6 & 10 & \\
7 & 60 & \\
\hline
\end{tabular}

each menus was progressively introduced. As described above, each time-out punishment was notified by a 3 -sec tone and the extinction of the house light for the whole duration of the punishment, which termination was signaled by house light illumination. Animals were first exposed to concave exposures, having only access to vulnerable and fragile holes during four 20-min sessions. They were then exposed to convex losses (only Anti-fragile and Robust holes available) for another four 20-min sessions. Thus, at the end of the training, all animals experienced both extreme events at least four times.

6. Final tests: For the final tests, animals were free to explore all menus throughout the forty 20-min sessions. Animals experienced four times the ten different sequences, randomly distributed across sessions. Population $(\mathrm{n}=20)$ was subdivided into two groups that experienced different, but equivalent, sequence-type distribution (Supplemental Figure 7)).

Figure 5 depicts a simplified time-line of the experimental procedure.

Figure 6 shows the chain of events in the ten sequences used for behavioral training and testing. Numbers in the second column indicate which event would occur and the sign preceding it whether it belongs to the gain (no sign) or loss (minus sign) domains. For example, the third event in the first sequence (noted -2) triggers a time-out punishment of 9 or $12 \mathrm{sec}$, depending on whether an animal performed his third nose poke in concave or convex menu, respectively. Note that extreme events (which should be noted 4 or -4 ) do not appear in the sequences, as they would automatically replace the 10th or 60th events of the sequence.

In Table 1 we report the different types of sequences used for behavioral training and testing, which shows the position of the extreme events. For example, in the sequence-type 6, jackpot could be obtained at the 10th position while the Black-swan would be triggered at the 60 th activation.

Figure 7 shows the different behavioral sequence-types used for the forty testing sessions. As indicated in the text, half of the population was subjected to the sequence-types described in the left part of the 'type 
column', while the other half experienced the sequence-types described in the right part of the 'type column'.

\section{A.3 Modelling and Statistical Analysis}

\section{Modelling Exposures}

Central to our experimental design is the notion of convex/concave exposure under radical uncertainty, that is, when probabilities and consequences are unknown a priori to subjects. A well known measure of convexity is the Jensen gap that is derived from Jensen's inequality ${ }^{6}$, which we now define and relate to statistical moments. In our context, the relevant form of Jensen's equality states, loosely speaking, that the expectation of a convex function of a random variable is larger that the value of that function when evaluated at the expectation of the random variable. Jensen's gap is then defined as the difference between the expectation of the function minus the value of the function at the expectation. The inequality is reversed for a concave function and the Jensen's gap is then defined as the difference between the value of the function at the expectation minus the expectation of the function.

In the gain domain, rats obtain $1,3,12$ or 80 sugar pellets if the convex exposure is chosen, or $2,4,5,5$ pellets if the concave exposure chosen. In the loss domain, convex exposure imposes $6,12,15$ or 15 seconds of time-out punishment, and 3, 9, 36, 240 seconds for the concave exposure. Because the values in the loss domain are proportional to the values in the gain domain, the former corresponding to 3 times the latter, we focus here on values for pellets. The statistical properties of convex and concave losses follow accordingly. More formally, sequences of gains are ordered by increasing values and are denoted $\left\{x_{i}^{\mathcal{X}}\right\}_{i=1}^{4}=\{1,3,12,80\}$ for the convex exposure and $\left\{x_{i}^{\mathcal{V}}\right\}_{i=1}^{4}=\{2,4,5,5\}$ for concave gains. We assume identical probabilities $\left\{p_{i}\right\}_{i=1}^{4}=\left\{p_{1}, p_{2}, p_{3}, \varepsilon\right\}$ for both concave and convex gains, where $\varepsilon$ is the ex-ante probability of the rare and extreme event (REE for short). The third value (that is, 12 for convex gains and 5 for concave gains) is labeled a rare event (RE), and the sets of the lowest two values (that is, 1 and 3 for convex gains, 2 and 4 for concave gains) for both exposures are composed of normal events (NE).

Making use of the exponential transform, we define Jensen gaps for the convex and concave exposures as, respectively, $\mathcal{J} \mathcal{G}_{\mathcal{X}}=\sum_{i=1}^{4} p_{i} e^{x_{i}^{\mathcal{X}}}-e^{\sum_{i=1}^{4} p_{i} x_{i}^{\mathcal{X}}}, \mathcal{J G} \mathcal{V}=e^{\sum_{i=1}^{4} p_{i} x_{i}^{\mathcal{V}}}-\sum_{i=1}^{4} p_{i} e^{x_{i}^{\mathcal{V}}}$, which relate to statistical moments as follows. The moment generating function for the convex and concave exposures are given by, respectively: $M_{\mathcal{X}}(t)=\sum_{i=1}^{4} p_{i} e^{t x_{i}^{\mathcal{X}}}$ and $M_{\mathcal{V}}(t)=\sum_{i=1}^{4} p_{i} e^{t x_{i}^{\mathcal{V}}}$. The series expansion of the exponential function allows us to derive:

$$
\begin{aligned}
& M_{\mathcal{X}}(1)=1+m_{1}^{\mathcal{X}}+\frac{1}{2 !} m_{2}^{\mathcal{X}}+\frac{1}{3 !} m_{3}^{\mathcal{X}}+\cdots+\frac{1}{N !} m_{N}^{\mathcal{X}}+\ldots \\
& M_{\mathcal{V}}(1)=1+m_{1}^{\mathcal{V}}+\frac{1}{2 !} m_{2}^{\mathcal{V}}+\frac{1}{3 !} m_{3}^{\mathcal{V}}+\cdots+\frac{1}{N !} m_{N}^{\mathcal{V}}+\ldots
\end{aligned}
$$

where $m_{N}^{\mathcal{X}}$ and $m_{N}^{\mathcal{V}}$ are the $N$-th statistical moments of the convex and concave exposures, respectively. For example, $m_{N}^{\mathcal{X}}=\sum_{i=1}^{4} p_{i}\left(x_{i}^{\mathcal{X}}\right)^{N}$ for any integer $N \geq 1$. Jensen's gaps for both exposures are then given by $\mathcal{J G}_{\mathcal{X}}=M_{\mathcal{X}}(1)-e^{\sum_{i=1}^{4} p_{i} x_{i}^{\mathcal{X}}}, \mathcal{J G}_{\mathcal{V}}=e^{\sum_{i=1}^{4} p_{i} x_{i}^{\mathcal{V}}}-M_{\mathcal{V}}(1)$. It follows that both Jensen's gaps, that are positive by definition, can be written in terms of moments.

More specifically, we set probabilities to $\left\{p_{i}\right\}_{i=1}^{4}=\{0.5-\varepsilon / 3,0.4-\varepsilon / 3,0.1-\varepsilon / 3, \varepsilon\}$ identically for both concave and convex gains. For convenience probabilities sum up to one, which means that in this section $\varepsilon$ is scaled up (that is, multiplied by two) compared to its magnitude in Section 2.1. Treating the probability for

\footnotetext{
${ }^{6}$ See [11] for a graphical exposition.
} 
Table 2: Jensen gaps and statistical moments for convex and concave exposures

$\begin{array}{lcc} & \text { Convex Exposure } & \text { Concave Exposure } \\ \log (\mathcal{J G}) & 75.682 & 2.880 \\ \text { Mean } & 3.895 & 3.118 \\ \text { Std Deviation } & 9.379 & 1.146 \\ \text { Skewness } & 7.169 & 0.203 \\ \text { Kurtosis } & 57.851 & 1.381\end{array}$

the REE as a varying parameter, it can be shown that Jensen's gaps for convex and concave exposures are ordered, with the former larger than the latter when $\varepsilon \leq 0.02$. Over that range, it also holds true that both Jensen's gaps are monotone increasing function of $\varepsilon$. While this property also holds for the expectations and variances of both exposures, it doesn't for higher-order moments. For example, the skewness and kurtosis of the convex exposure turn out to be hump-shaped functions of $\varepsilon$, with peaks corresponding to values of $\varepsilon$ smaller than one percent. This fact underlines that convexity measured by Jensen's gap offers a unifying approach to rank exposures, seen as lotteries, whereas statistical moments do not necessarily do so. ${ }^{7}$ In Table 2 we report the (log of) Jensen gaps and the moments for both exposures when $\varepsilon=1 / 75 \approx 1.3 \%$, which corresponds to obtaining one REE out of 75 nose pokes.

We derive next the ex-ante properties of the probability distributions associated with concave and convex gains: ${ }^{8}$

- In the domain restricted to NE, concave gains first-order stochastically dominate convex gains. In addition, concave gains then have a larger expected value than that of convex gains, with equal variance, skewness and kurtosis for concave and convex exposures.

- In the domain restricted to $\mathrm{NE}$ and $\mathrm{RE}$, concave gains second-order stochastically dominate convex gains. In addition, concave gains then have a larger expected value and smaller variance, skewness and kurtosis than that of convex gains.

- In the full domain including NE, RE and REE, convex gains second-order stochastically dominate concave gains if and only if $\varepsilon \geq 0.302 \%$. In addition, convex gains then have a larger expected value, variance, skewness and kurtosis than that of concave gains if $\varepsilon \geq 0.302 \%$.

The above assumptions on the experimental design are stated in terms of stochastic dominance and moments of the probability distributions. They also have implications in relation to standard approaches to decisionmaking. First, from an ex-ante perspective with perfect information about the probability distributions of all exposures, value-maximizing subjects whose preferences are represented by any non-decreasing value function would choose concave gains in the domain restricted to NE. They would continue to do so in the domain restricted to NE and RE for any non-decreasing and concave value function. However, in the full domain with REE, value-maximizing subjects endowed with any non-decreasing and concave value function

\footnotetext{
${ }^{7}$ We conjecture that, more generally, all lotteries satisfying our assumptions (including monotone probability distribution) can be ranked according to their convexity as measured by Jensen's gap.

${ }^{8}$ For definitions of stochastic dominance and congruent utility classes, see for example Fishburn and Vickson [6]. Also note that first-order (resp. second-order) stochastic dominance implies second-order (resp. third-order) stochastic dominance.
} 
are predicted to choose convex gains.

We denote $\mathbb{V}\left(\left\{x_{i}\right\}_{i=1}^{n},\left\{p_{i}\right\}_{i=1}^{n}\right)$ the value attributed to sequences of gains $\left\{x_{i}\right\}_{i=1}^{n}$ with probabilities $\left\{p_{i}\right\}_{i=1}^{n}$, for $n \leq 4$. The above assumptions have the following implications in terms of value maximization:

- In the domain restricted to $\mathrm{NE}, \mathbb{V}\left(\left\{x_{i}^{\mathcal{V}}\right\}_{i=1}^{2},\left\{p_{i}\right\}_{i=1}^{2}\right)>\mathbb{V}\left(\left\{x_{i}^{\mathcal{X}}\right\}_{i=1}^{2},\left\{p_{i}\right\}_{i=1}^{2}\right)$ holds.

- In the domain restricted to $\mathrm{NE}$ and $\mathrm{RE}, \mathbb{V}\left(\left\{x_{i}^{\mathcal{V}}\right\}_{i=1}^{3},\left\{p_{i}\right\}_{i=1}^{3}\right)>\mathbb{V}\left(\left\{x_{i}^{\mathcal{X}}\right\}_{i=1}^{3},\left\{p_{i}\right\}_{i=1}^{3}\right)$ holds.

- In the unrestricted domain, $\mathbb{V}\left(\left\{x_{i}^{\mathcal{X}}\right\}_{i=1}^{4},\left\{p_{i}\right\}_{i=1}^{4}\right)>\mathbb{V}\left(\left\{x_{i}^{\mathcal{V}}\right\}_{i=1}^{4},\left\{p_{i}\right\}_{i=1}^{4}\right)$ holds if and only if $\varepsilon \geq$ $0.302 \%$.

Note that the reversal in second-order stochastic dominance (and value) in the full domain favors convex gains when extreme events that are indeed very rare - with probabilities much smaller that one percent are added. In contrast, absent REE, adding only a RE is not enough to favor convex gains, even though it allows subjects to possibly detect convexity/acceleration and concavity/deceleration of gains and losses.

The above assumptions hold true under expected utility, that is when $\mathbb{V}(\cdot, \cdot)=\sum_{i=1}^{n} p_{i} u\left(x_{i}\right)$ with the appropriate conditions on the utility function $u$ (non-decreasing for first-order stochastic dominance, nondecreasing and concave for second-order stochastic dominance; see [6]). To better match experimental data, however, expected utility is increasingly supplemented by some form of probability weighting $w\left(p_{i}\right)$. For instance, [4] use a two-parameter functional form due to [13] and find that 30 rats out of 36 behave as if their probability weighting $w\left(p_{i}\right)$ is concave. In that case, the above ex-ante properties hold mutatis mutandis, for example, under expected utility with probability weighting, defined as $\sum_{i=1}^{n} w\left(p_{i}\right) u\left(x_{i}\right)$. Alternatively, in the setting of rank-dependent expected utility, we can make us of results 3 and 4 in [9] to show that the above ex-ante properties still hold provided that the transformation of the cumulative distribution function is any increasing-concave function. Note that since in our experimental design REEs are both extremes (in the sense of being the largest values) and rare (that is, they have very low probabilities), one expects similar results under probability weighting and rank-dependent expected utility (or cumulative prospect theory for that matter). Although in theory assuming simply a weighting function $w\left(p_{i}\right)$ that is inverse-s-shaped and "very" convex for large gains (see [13] for the associated parametric restrictions) could overturn the rankings of concave and convex exposures stated above, results in [4] suggest that this is not to be expected for the overwhelming majority of the rats that are subject to their experiments.

\section{Statistical Analysis}

Statistical test for correlation TSREE/OSREE for in panel $(c)$ of Figure 2 and for mean difference before/after here.

\section{References}

[1] Bechara A, Damasio AR, Damasio H, Anderson SW (1994) Insensitivity to future consequences following damage to human prefrontal cortex. Cognition 50: 7-15. 2

[2] Benartzi S., and Thaler R.H. (2001). Naive diversification strategies in defined contribution saving plans. The American Economic Review, 91: 79-98 6 
Table 3: Mean difference for each of the 20 rats before/after

\begin{tabular}{|c|c|c|c|c|}
\hline \multirow[b]{2}{*}{ Rat } & \multicolumn{2}{|c|}{ TSREE } & \multicolumn{2}{|c|}{ BSA \& JS } \\
\hline & jackpot & black swan & jackpot & black swan \\
\hline 1 & -0.062 & -0.133 & -0.046 & -0.467 \\
\hline 2 & -0.025 & -0.108 & -0.075 & 0.215 \\
\hline 3 & 0.050 & 0.050 & -0.100 & -0.083 \\
\hline 4 & -0.036 & 0.100 & -0.036 & -0.300 \\
\hline 5 & 0.010 & 0.400 & 0.010 & -0.067 \\
\hline 6 & 0.100 & 0.117 & -0.100 & 0.017 \\
\hline 7 & -0.650 & 0.186 & 0.050 & 0.000 \\
\hline 8 & 0.017 & 0.111 & -0.050 & -0.044 \\
\hline 9 & -0.264 & -0.210 & 0.009 & -0.070 \\
\hline 10 & -0.045 & 0.400 & -0.009 & 0.200 \\
\hline 11 & -0.175 & 0.080 & 0.042 & -0.027 \\
\hline 12 & 0.033 & 0.150 & -0.122 & 0.000 \\
\hline 13 & 0.085 & 0.150 & 0.054 & -0.350 \\
\hline 14 & -0.114 & -0.100 & -0.086 & -0.500 \\
\hline 15 & 0.167 & 0.317 & -0.011 & -0.050 \\
\hline 16 & -0.000 & -0.100 & -0.062 & -0.100 \\
\hline 17 & 0.300 & 0.150 & 0.100 & -0.107 \\
\hline 18 & -0.190 & 0.600 & -0.010 & 0.000 \\
\hline 19 & 0.105 & 0.160 & -0.053 & -0.040 \\
\hline 20 & 0.014 & -0.100 & -0.029 & -0.300 \\
\hline
\end{tabular}

Table 4: Ex-Post Outcomes for each of the 20 rats - Summary Statistics

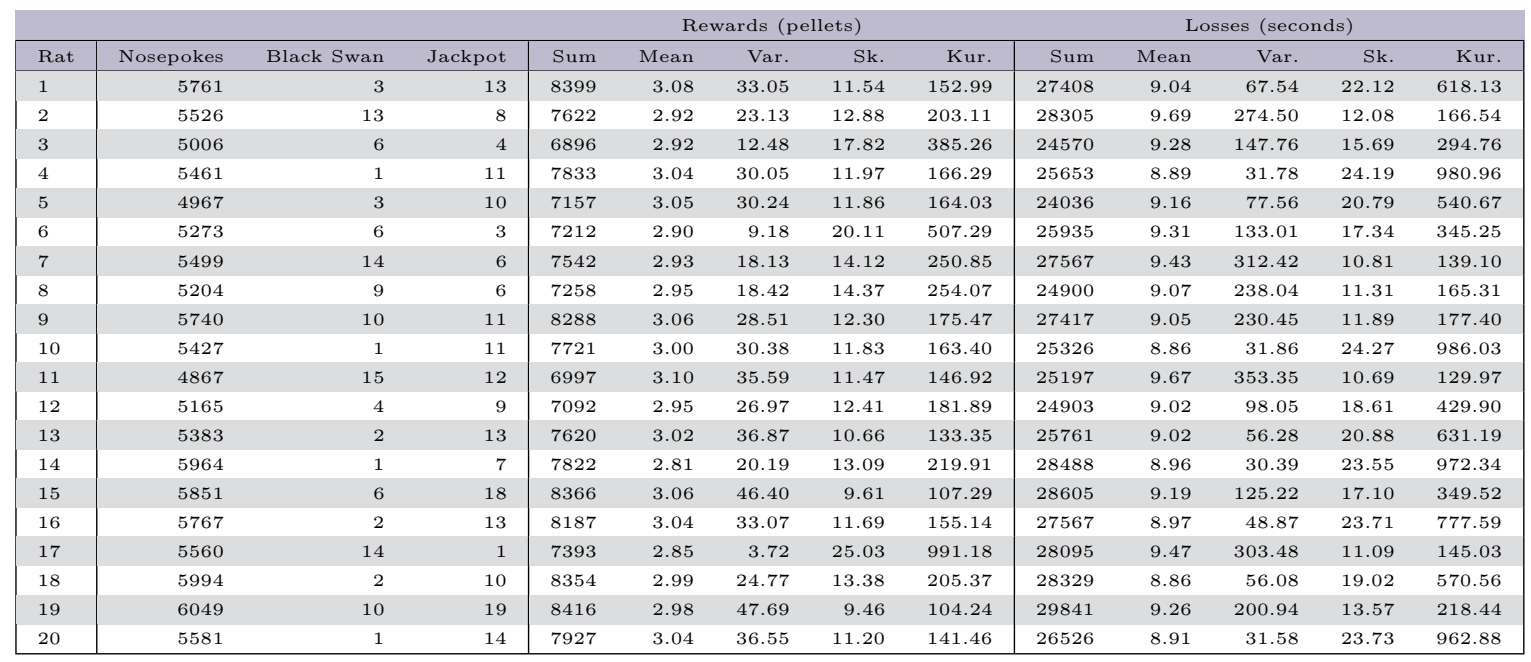


[3] Cinotti, F., Fresno, V., Aklil, N., Coutureau, E., Girard, B., Marchand, A. R., and Khamassi, M. (2019). Dopamine blockade impairs the exploration-exploitation trade-off in rats. Nature Scientific Reports, 9: 6770. 2

[4] Constantinople,C. M., Piet, A. T., and Brody, C. D. (2019). An analysis of decision under risk in rats. Current Biology, 29: 2066-2074. 2, 4, 12, 19

[5] Douglas R.J. (1989). Using SAB as a tool: advice from a veteran. In "Spontaneous Alternation Behavior", W.N. Dember and C.L. Richman, eds. Springer, New-York, 145-59. 12

[6] Fishburn, P. C., and Vickson, R. G. (1978). Theoretical foundations of stochastic dominance. In "Stochastic Dominance", G. A. Whitmore and M. C. Findlay, eds. D. C. Heath and Co., Lexington, Massachusetts, 37-113. 18, 19

[7] Hertwig, R., and Erev, I. (2009). The description-experience gap in risky choice. Trends in Cognitive Sciences, 13: 517-523. 12

[8] Kalenscher, T., and M. van Wingerden (2011). Why we should use animals to study economic decision making - a perspective. Frontiers in Neuroscience, 5: 1-8. 2

[9] Loehman, E. (1994). Rank dependent expected utility: stochastic dominance, risk preference, and certainty equivalence. Journal of Mathematical Psychology, 38: 159-197. 19

[10] Mehlhorn, K., Newell, B. R., Todd, P. M., Lee, M. D., Morgan, K., Braithwaite, V. A., Hausmann, D., Fiedler, K., and Gonzalez, C. (2015). Unpacking the exploration-exploitation tradeoff: a synthesis of human and animal literatures. Decision, 2: 191-215. 2

[11] Needham, T. (1993). A visual explanation of Jensen's inequality. The American Mathematical Monthly, 100: 768-771. 17

[12] Pittaras E, Cressant A, Serreau P, Bruijel J, Dellu-Hagedorn F, Callebert J et al (2013) Mice gamble for food: individual differences in risky choices and prefrontal cortex serotonin. Journal of Addiction Research and Therapy S4:011. 2

[13] Prelec, D. (1998). The Probability weighting function. Econometrica, 66:497-527. 19

[14] Real, L.A (1980). Fitness, uncertainty, and the role of diversification in evolution and behavior. The American Naturalist, 115: 623-638. 6

[15] Real, L.A. (1991). Animal choice behavior and the evolution of cognitive architecture. Science, 253: 980-986. 2

[16] Rees, M., Jessica, C., Metcalf, E., and Childs, D. Z. (2010). Bet-hedging as an evolutionary game: the trade-off between egg size and number. Proceedings. Biological sciences, 277: 1149-1151. 6

[17] Simons A. M. (2011). Modes of response to environmental change and the elusive empirical evidence for bet hedging. Proceedings. Biological sciences, 278: 1601-1609 6

[18] Taleb, N. (2012). "Antifragile, things that gain from disorder". Random House. 2, 4

[19] Teodorescu, K., and Erev, I. (2014). On the decision to explore new alternatives: the coexistence of under- and over-exploration. Journal of Behavioral Decision Making, 27: 109-123. 2

[20] de Visser L, Homberg JR, Mitsogiannis M, Zeeb FD, Rivalan M, et al. (2011) Rodent versions of the iowa gambling task: opportunities and challenges for the understanding of decision-making. Frontiers in Neuroscience 5: 109. 2 
Figure 6: Succession of events for each sequence

\begin{abstract}
\# Succession of events
$12,-1,-2,-2,2,1,2,-1,2,1,-2,1,2,-1,-3,1,-1,-2,-2,-1,1,1,3,1,1,1,1,-1,1,-2,2,-1,-2,1,-3,-1,2,-1,-2,-1,-1,1,2,-1,-3,1,-3,-1,-2,-1, \quad-2, \quad 1$, $2,2,-1,-1,-2,1,2,1,-1,1,2,-3,-2,-1,-1,-1,-1,-1,-3,-1,-2,-1,-2,-1,-2,-1,3,-2,2,2,-1,1,-1,1,-2,1,-2,1,-2,1,2,1,-1,-2,1,1,1,-1,1,2,2,-1$, $1,-1,2,-1,1,-1,-2,2,2,1,1,-1,1,1,-2,1,3,1,-1,1,1,1,-2,1,-2,1,-2,-1,2,-1,2,-1,-1,-1,1,-1,1,-1,2,1,-2,1,-2,-1,2,-1,-1,-1,-1,1,-2,1,-1$, $-1,-3,2,2,2,2,-1,-2,-1,-2,1,3,1,2,1,-1,1,2,-1,-3,1,2,1,-2,-2,1,1,-3,-1,1,1,-2,1,2,2,-3,1,1,-1,3,-2,-2,1,2,-1,2,1,-2,1,-1,2,-1,-1,-3$ $2,2,-2, \quad 2,1,-1,-1,2,-1,-2,1,2,-1,3,1,1,1,-2, \quad 2,-3,1,-2,1,2,1,-1,-1,2,1,3,-1,-3,1,-1,1,1,-1,-1,-2,-3,-1,-3,1,1,1,-1,1,-2,-1,3,-1, \quad 1$, $1,-2,2,-3,-1,1,1,2,-1,-2,1,2,1,3,-1,-2,2,1,2,-1,1,-1,1,2,-1,3,-1,-2,-2,-1,-1,-1,-1,1,-1,1,1$

$2-2,1,-1,-2,-1,-1,-3,1,2,1,-2,2,2,1,1,2,-2,-1,3,-2,1,1,-1,2,2,1,-3,-1,-2,-1,-2,1,-2,1,-1,1,-2,-2,1,1,-2,-1,-2,-2,-2,1,2,2,1,1,-2,-1$, $1,-1,-1,1,-2,1,-2,1,-2,2,2,1,1,1,1,-1,-1,1,-1,1,-2,2,-3,-2,-3,-1,1,1,-2,-1,1,-1,-2,1,-3,1,-1,1,-1,-1,-2,-1,3,-2,-1,1,2,-1, \quad 2, \quad 1,1$, $1,3,-1,2,-1,2,-1,-1,-1,3,1,-3,1,2,-1,2,-1,1,1,-1,-1,-2,-1,-3,-1,-1,-1,-2,-1,-2,-1,-2,1,3,1,-2,1,-2,2,-3,-1,-2,2,1,2,-3,1,3,1, \quad-2, \quad 1$, $2,2,-1,-1,3,-1,-2,1,-3,-1,2,1,2,1,1,1,-1,1,-1,-1,3,-1,2,-1,-3,-2,3,1,-1,-2,2,1,2,-1,-2,-1,2,1,1,-1,1,-1,-2,-1,1,-1,1,-1,1,-1,1,-2,--$ $2,1,1,1,-2,1,2,1,1,-1,-2,-1,1,2,-2,-1,2,-1,-2,-2,-2,-1,-2,1,2,-1,2,-2,-1,1,3,-1,-3,1,-1,1,-3,1,-3,-2,3,1,2,-1,3,-1,3,-1,-3,1,-1,-1$, $2,2,1,1,-1,-1,-2,-1,-2,1,2,-1,-1,-1,3,2,-2,1,-2,-1,-1,1,-2,2,3,1,-2,1,2,-1,-2,-1,-1,-1,2,-1,-1,-2,-1,-1,-3,1$
\end{abstract}

$3-2,1,-2,-1,-2,-1,-2,-1,-3,-1,1,1,2,-1,2,1,2,1,-3,-1,-2,-1,1,1,2,-2,-2,-1,2,-1,-2,-3,3,-1,1,-1,1,2,1,1,-1,-1,-1,1,-1,-1,-2,-2,-1,-1$, $2,1,3,-1,-2,1,-2,1,-1,1,-2,1,2,-1,2,1,-2,1,2,-1,1,-1,-2,-1,-3,1,-2,1,-2,1,-2,-1,2,1,2,-1,-2,1,-1,-1,-2,-1,3,1,-2,-1,3,-1,-1,-1,-3$, $-1,-2,2,1,2,1,-1,1,-1,-2,-1,2,-2,-1,1,1,1,-1,-1,-2,-2,3,1,-3,2,2,2,-1,-1,-2,-1,-2,1,-1,1,2,-1,-2,-1,-1,1,1,1,-1,-2,2,1,2,1,-1,1,1$, $-1,-1,-1,2,1,2,1,2,-1,-1,-1,2,-1,-1,1,2,-2,-2,1,-1,1,1,-2,-1,-1,2,-1,-3,-1,3,-2,-1,1,-1,-1,-1,2,-2,-2,-2,-1,-1,-1,1,-1,-1,1,1,-1,2$, $1,1,1,2,-1,-2,1,1,-1,1,-1,-2,1,1,1,2,-2,3,-3,1,-1,-3,-1,1,-2,-1,1,1,-2,1,-1,3,1,-1,-1,-2,-1,2,-2,2,1,3,1,1,1,-3,-2,-1,1,-2,1,3,-1$, $-1,-1,-2,2,2,-1,2,1,2,-1,2,1,-1,-1,1,-2,1,1,2,-1,2,1,-3,1,3,1,2,-1,-2,-1,-3,1,-2,2,-3,1,1,-1,3,1,-1,2,1,1$

$41,-1,-2,2,-1,1,-2,1,-2,1,-3,-1,-2,2,-1,1,2,1,2,-2,-2,1,-2,-2,2,1,-1,-1,-2,-1,-1,-1,-1,2,-1,-1,-2,2,3,-1,-1,1,-2,-1,2,-1,1,-1,-2,1,-$ $2,-1,1,-1,2,1,2,1,2,-1,-3,1,-1,1,-2,-1,-3,-1,1,-1,2,2,-3,-1,1,-2,-1,-1,-1,1,2,-1,2,-1,-3,-1,-1,-1,-2,1,3,1,-2,-2,1,-2,2,1,-3,-1,1,-$ $1,-3,1,-2,1,2,-1,-1,-1,1,-1,-3,1,-2,-2,-3,-2,-2,1,-2,-1,-2,-1,-2,1,1,1,-2,1,2,1,-2,1,2,-1,-1,1,-1,-1,-3,1,-2,-1,-2,-1,-2,-1,1,1,-2$, $1,1,-2,2,-1,-1,1,-3,-2,-3,1,-2,1,2,-1,2,2,1,2,-1,-1,3,2,-2,-1,2,-2,-2,-1,2,-2,-1,1,2,-1,1,-1,1,-1,-2,-1,-2,1,3,-2,-3,1,-3,-1,1,1,3,1,-$ $2,-1,-1,-2,-2,1,2,-2,-2,-1,1,1,2,-1,-2,1,3,1,2,1,-1,-1,-2,1,1,-2,1,1,-1,-1,-1,-1,2,1,-2,1,1,-1,2,1,2,-1,1,2,-2,1,3,1,1, \quad-2,-2,1,-$ $1,2,1,-1,-2,-1,-2,1,-2,-1,-3,2,-2,1,-1,-1,-2,-1,-3,2,-2,-1,1,-1,2,-2,-3,-1,3,-2,1,1,-1,-1,1,1,2,1,2,-1,2,1,-1,1$

$5 \quad 1,-1,2,2,3,-1,2,-1,2,1,-2,1,2,-1,-1,-1,-2,-1,-1,2,-3,-1,-1,3,2,1,2,-1,1,1,-2,-1,2,-2,1,1,-2,-1,-2,1,-2,-1,-2,1,1,-1,-3,1,1,-1,-1,-2,2,-$ $1,-2,-2,-2,1,-1,1,-1,-1,2,-1,2,1,-1,2,-2,1,2,-1,2,1,2,-2,-2,1,1,1,-2,-1,-2,-1,-2,-2,-1,2,3,1,1,-1,-2,1,1,-1,2,1,2,1,-3,1,2,-1,2,-1,-$ $2,1,2,1,-1,-2,-1,-1,2,1,2,2,-2,1,2,-1,-2,-1,-3,-1,-1,-1,-1,1,2,-1,-1,-3,2,1,-1,-1,3,1,-1,1,1,-1,3,-1,-2,1,-1,-1,3,1,2,1,-1,1,2,-1,2,1,-$ $3,1,-2,1,-2,1,2,1,-1,-2,-1,-2,-2,-1,2,1,2,1,-1,-1,2,-2,2,-1,-3,1,1,1,3,-2,-3,-1,3,1,-1,1,2,1,1,1,1,2,2,1,2,-1,2,1,-3,-1,2,-1,1,-1,-$ $2,1,1,1,-1,-2,-1,2,-1,-1,-2,1,2,1,2,-1,2,-1,2,-2,-1,-1,-1,-2,1,1,-2,-1,-2,1,-2,1,-1,2,3,1,1,-1,-2,-2,-2,1,-2,1,2,-1,3,2,-2,2,-2,1,1,1,-$ $2,-2,3,1,-2,-1,-3,-1,3,-1,-2,-1,2,-2,-3,1,-3,-1,1,1,1,1,-2,-1,-3,-1,-1,-2,3,1,1,-1$

$6 \quad-1,1,1,-1,-2,1,-2,-1,-2,2,-1,1,-2,-1,2,1,1,1,-2,-1,1,1,2,-2,2,1,3,-1,2,-1,-3,-1,-2,1,-2,-1,-2,-1,-1,1,-1,1,-3,2,-1,-2,1,1,-1,1,-$ $2,1,1,1,1,1,1,2,-2,1,-2,2,-2,-1,1,1,-1,-1,-1,1,3,2,-2,-1,3,-1,-1,-1,1,1,-1,-2,2,1,-1,-1,-3,-1,-2,-1,-2,1,1,-2,-2,1,-2,1,2,1,3,1,1,-$ $1,1,-1,-1,-1,2,-2,3,-2,2,-3,2,1,-1,2,-3,1,3,1,-1,1,-2,-1,1,-1,-1,-1,2,2,-2,-2,1,-1,1,1,1,-1,2,2,1,-1,1,1,-2,1,3,-2,1,1,3,-1,-3,-1,1,1,-$ $3,1,3,1,1,-1,-2,-1,2,-1,-1,1,2,-1,1,-1,-1,-1,-2,-1,-2,-1,-3,-1,2,1,-1,-1,2,2,-2,-1,-2,-1,3,1,-2,-1,-1,1,3,-2,-2,-2,-2,-1,-2,2,-2,-1,1,-$ $1,-2,1,1,-1,-3,-1,-2,1,3,1,-2,-1,-3,1,1,1,-2,-1,1,-1,-2,-1,-1,-1,-1,-1,2,1,1,-2,1,1,1,-2,-1,-1,-2,1,2,-2,3,-1,-3,-1,1,1,-2,1,3,2,1,1,-$ $2,-2,-2,1,2,1,2,-1,-1,-1,2,1,-1,-1,1,-1,1,-1,-1,-1,-2,-1,1,1,-2,-1,-1,2,-2,-2,1,1,-2,1,-2,1,2,1$

$7 \quad-2,-1,-2,1,2,1,-2,-2,1,-1,-1,-1,-3,1,-1,-1,-1,1,2,1,2,-1,-2,-1,-1,1,-2,1,2,1,-2,-1,3,-1,2,-1,-3,1,-2,-1,-2,2,3,-1,-3,1,-2,2,1,1,2,1,-$ $2,-1,-2,1,3,-1,-1,1,-1,1,3,-3,-3,-1,2,-1,-1,-2,2,1,1,-1,-2,-1,1,1,-2,-1,-2,-1,2,-2,-1,-1,2,-1,2,1,-1,1,2,1,2,1,-2,-2,-1,-1,-1,1,-2,--$ $1,2,1,-2,-2,-1,1,2,2,-2,-1,2,1,2,1,2,1,-2,1,-1,1,2,-1,2,-1,3,1,-2,1,2,1,-2,-1,-2,1,-3,-2,-3,1,1,-1,-2,1,-2,-2,-3,2,2,2,-2,-1,1,1,-2,1,-$ $1,-1,-2,1,-3,1,3,-1,-3,-1,2,1,-3,-2,-2,1,2,1,2,-1,-2,1,-3,1,-2,1,3,1,2,2,2,-2,2,-1,-2,1,3,-1,1,1,-2,-1,3,1,-1,1,-3,1,-2,-1,-3,-2,-1,-$ $2,2,1,1,1,-3,-1,2,-1,-1,2,-1,1,-2,-1,1,1,-1,1,2,-1,3,1,1,-1,2,2,-2,-1,-2,1,1,-2,-2,2,2,-1,1,-1,-2,-1,-2,1,1,-2,-2,1,2,-1,2,1,1,-2,1,-$ $1,-2,1,-1,-1,1,-2,-2,-1,-2,1,2,1,2,1,1,-1,2,-1,-1,2,3,-1,-1,2,1,2,-1,1,-1,-1,-3,1,2,-2$

$8-1,1,-2,-1,-2,-1,-1,1,-2,-1,2,-1,3,1,-2,-1,-2,1,-3,-1,2,-1,3,1,-2,1,-1,1,-2,-1,2,2,-1,-1,-2,1,2,-1,-3,1,-2,1,1,-1,2,1,1,2,-3,-2,2,1,-$ $3,-1,-1,1,1,1,2,1,-1,1,2,-1,-1,1,2,-1,3,2,-1,-1,-1,1,3,-1,-2,1,-2,-1,-1,-2,2,-1,1,-1,1,-3,-1,2,2,1,2,1,-2,1,-1,1,-1,1,2,1,3,1,3,-1,-$ $1,-1,-3,1,-2,-1,-1,1,1,-1,2,1,-2,-1,-1,1,-2,1,-1,1,2,1,-2,-1,-2,1,-2,-1,-1,-1,2,1,-1,-1,2,2,2,1,-2,2,1,1,-3,-1,1,-1,-1,-1,1,-1,-1,-$ $1,2,-1,-2,1,1,-1,2,1,-2,1,1,1,1,-1,1,-1,3,2,-1,-1,-2,1,-1,-2,1,1,1,1,-3,1,-1,2,-2,1,-2,-2,3,-1,-3,1,1,1,-2,1,-2,1,-1,-1,1,1,-2,1,-3,--$ $1,2,1,-1,1,2,-1,-3,1,2,-1,-2,2,2,-2,-1,1,-1,1,1,1,-2,1,2,-1,-1,1,2,-1,2,-2,-2,-1,-2,1,-2,1,2,2,-2,-1,-3,-1,2,1,1,-1,1,-3,-1,-1,-1,1,-$ $1,-1,3,1,-2,-2,-1,1,-2,1,-1,-2,2,1,2,1,-3,-1,-3,1,-2,-1,3,-1,3,-2,-3,1,-2,-1,1,-1,-1,1,1,1$

$91,1,2,-1,-2,1,1,-1,-2,1,1,1,1,-1,-1,-1,-2,-1,-2,1,2,-2,-2,1,1,-2,1,-1,-3,2,-1,1,3,-2,-3,-1,2,1,-2,1,3,2,-2,1,-2,1,-3,1,2,1,-1,1,2,-$ $1,3,-1,-2,1,2,-2,-1,-1,3,1,2,-1,1,1,-1,-1,1,-1,-2,2,3,1,-2,-1,2,-2,2,1,-1,-1,2,-1,2,-1,2,1,-2,-1,3,-1,-2,2,2,2,1,1,1,2,3,-2,3,-1,-1,-$ $1,2,-1,1,-1,1,1,-1,-1,-3,1,-1,2,-1,-1,-3,1,-2,-1,-1,1,-2,-2,2,1,-2,-1,-2,2,-2,1,2,-2,1,1,-1,-1,-2,1,1,-2,-1,2,-3,-1,2,-1,-2,-1,2,-1,-$ $3,2,-1,1,2,-1,1,1,2,-1,-2,1,1,2,2,1,3,-1,1,1,-2,1,2,1,-1,-1,-2,-1,3,1,1,1,1,1,1,-1,-2,-1,-2,2,-3,1,1,-1,3,-2,3,-1,1,-1,-1,-2,-2,1,-$ $2,1,-1,-1,-2,1,-2,-2,2,-1,-1,1,-2,1,1,-1,1,2,1,-1,-1,1,-3,-1,-2,-1,-2,1,-2,-1,2,-1,-1,-1,-1,1,1,-1,-2,1,-2,-2,1,-1,2,-3,1,-1,-2,1,-$ $2,1,2,-1,-1,1,-2,-1,3,1,-3,1,2,1,1,1,-2,-1,-1,1,-3,-1,-1,-1,-3,2,3,-1,2,-2,3,-1,2,-1,2,-1,-2,-1$

$10-1,1,3,-1,-2,-1,-2,-1,3,1,2,1,2,-1,-2,1,-3,1,2,-1,-3,-2,2,-1,3,-2,2,-1,2,1,-2,-1,-2,1,3,2,2,-1,2,1,3,1,1,1,-1,-1,-1,1,-3,1,1,1,2,1,1,-$ $2,1,2,-1,-1,-2,-1,1,-1,2,1,-2,1,-2,1,-1,1,2,-2,-2,-1,-2,-1,-2,-1,-2,-1,2,-1,-1,-2,-1,-1,-3,1,-3,1,-1,2,-2,1,2,-1,-1,-1,1,1,-2,2,3,1,-$ $3,1,2,1,-1,1,2,-2,-1,-1,-2,1,1,-1,-1,1,-3,1,-3,2,-3,1,-1,1,-2,1,-2,1,2,-1,3,-1,2,-2,1,-1,2,2,-3,1,-2,2,2,-1,2,2,-2,-1,1,-1,2,1,-1,1,-$ $1,-1,-1,-1,2,1,1,2,1,1,3,1,3,1,-2,-2,-1,-1,-2,-1,-2,-1,3,-2,1,-1,-1,-1,-1,1,2,-1,2,1,3,-1,2,-2,2,-1,-3,-1,1,-2,1,-1,-3,1,1,-1,-2,1,1,-$ $1,-2,1,1,1,-1,-1,2,-2,-2,1,2,-2,3,1,-1,3,-3,1,2,-1,1,-1,-2,1,2,2,2,-2,-3,-1,-2,-1,2,1,-2,1,-2,1,3,1,-2,-1,2,-1,-2,-1,-1,-2,3,-1,1,-$ $2,2,-2,2,1,-1,2,-2,1,2,1,3,-1,1,-1,2,1,-2,-1,1,-2,1,-1,-1,-1,3,1,2,1,3,1,-3,-1,-1,1$ 
Figure 7: Sequences and types Session Sequence Type

\begin{tabular}{|c|c|c|}
\hline 1 & 2 & 5 \\
\hline 2 & 10 & 6 \\
\hline 3 & 5 & 1 \\
\hline 4 & 3 & 7 \\
\hline 5 & 4 & 3 \\
\hline 6 & 1 & 7 \\
\hline 7 & 6 & 2 \\
\hline 8 & 6 & 6 \\
\hline 9 & 4 & 2 \\
\hline 10 & 1 & 1 \\
\hline 11 & 7 & 7 \\
\hline 12 & 1 & 3 \\
\hline 13 & 7 & 7 \\
\hline 14 & 8 & 2 \\
\hline 15 & 5 & 2 \\
\hline 16 & 7 & 2 \\
\hline 17 & 3 & 1 \\
\hline 18 & 5 & 5 \\
\hline 19 & 2 & 1 \\
\hline 20 & 7 & 6 \\
\hline 21 & 9 & 4 \\
\hline 22 & 4 & 2 \\
\hline 23 & 2 & 5 \\
\hline 24 & 2 & 3 \\
\hline 25 & 4 & 2 \\
\hline 26 & 9 & 2 \\
\hline 27 & 10 & 5 \\
\hline 28 & 10 & 5 \\
\hline 29 & 10 & 1 \\
\hline 30 & 3 & 3 \\
\hline 31 & 8 & 5 \\
\hline 32 & 3 & 7 \\
\hline 33 & 6 & 2 \\
\hline 34 & 9 & 4 \\
\hline 35 & 9 & 3 \\
\hline 36 & 6 & 5 \\
\hline 37 & 1 & 5 \\
\hline 38 & 8 & 4 \\
\hline 39 & 5 & 3 \\
\hline 40 & 8 & 4 \\
\hline
\end{tabular}

